\title{
Workspace fixation for free-floating space robot operations
}

\author{
Alessandro M. Giordano ${ }^{a}$, Davide Calzolari ${ }^{a}$, Alin Albu-Schäffer ${ }^{a}$
}

\begin{abstract}
When a space robot accidentally or voluntarily comes in contact with a target object, a workspace shift happens due to exchange of momentum between the objects. The problem of workspace adjustment is addressed herein. A novel controller is derived to simultaneously adjust the workspace and control the end-effector pose. The controller is based on a center-of-mass (CoM) regulation which fixes the workspace in the inertial space while leaving the base free to move, resulting in fuel efficiency. The control is validated on hardware using a robotic simulator composed of a seven degree-of-freedom (DOF) arm mounted on a 6DOF moving base.
\end{abstract}

\section{INTRODUCTION}

The design of efficient control strategies for space robots is a key point for a sustainable development of orbital robotic systems. Improved coordination of satellite-arm motion would reduce fuel consumption of such systems, thus increasing the operational life or equivalently, decreasing the launch weight due to fuel load. The search for efficient space robot control systems in the past has led to the concept of free-floating robot, for which the satellite base is left free to move during robot maneuvers. According to this strategy, the satellite thrusters are completely turned off to conserve precious, nonrenewable fuel [1], [2].

Today, some limitations of a completely free-floating approach are recognized [3],[4],[5],[6]. Namely, any voluntary or accidental contact induces an inertial drift in the freefloating system with respect to the given target location. This risky situation can only be recovered by using external actuators to stop the drift and restore the proper robot-target location. For this purpose, in [5], the robot-target position is restored using a switching strategy. The robot is first operated in free-floating mode to achieve a desired impedance on the end-effector. Then the drift in the base-target position due to the contact is stopped by switching to a base position control strategy, which brings the base back to the desired position. A drawback of this approach is that, because the drift is relatively fast even for small contact forces, the strategy would require very frequent switching between controllers. In [3] a momentum-dumping strategy was derived to remove linear and angular drift. The inertial drift is stopped by dumping the accumulated momentum using external actuators. By exploiting the triangular structure of the momentum-arm dynamics highlighted therein, momentum dumping and endeffector control are accomplished simultaneously, thereby avoiding controller switching.

The strategy in [3] is effective for stabilizing the motion but

${ }^{a}$ The authors are with the DLR Institute of Robotics and Mechatronics, 82234 Weßling, Germany and with the Technical University of Munich, Garching, 85748, Germany. Contact alessandro.giordano@dlr.de
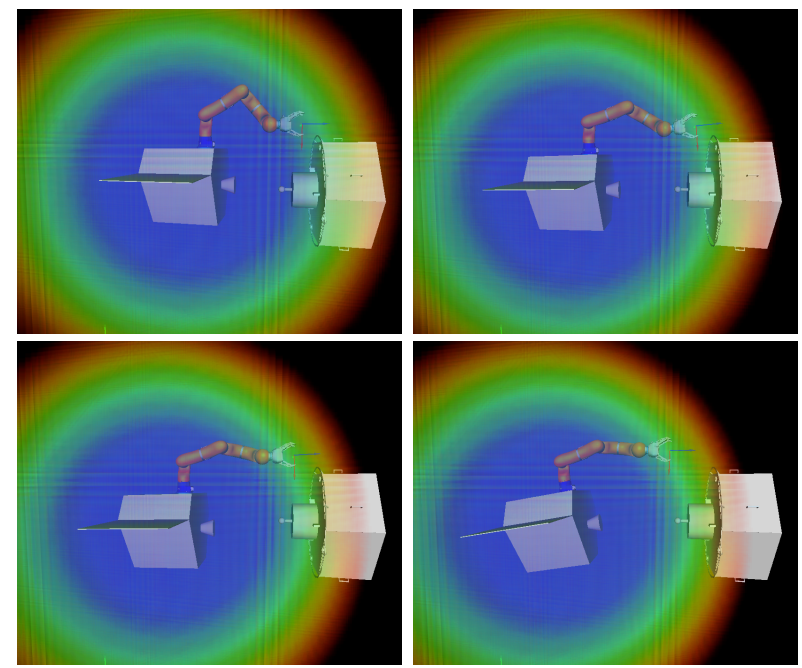

Fig. 1. Rendered workspace of multiple contacts experiment. The system CoM moves after each impulse and the target satellite exits the robot workspace.
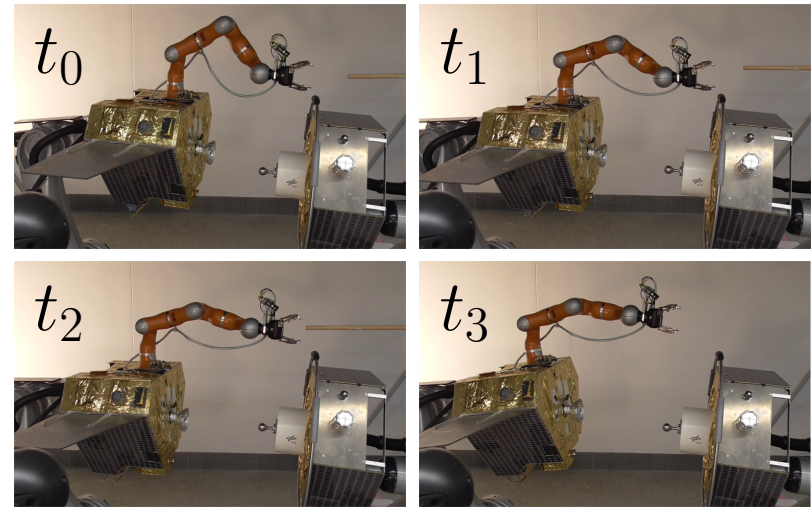

Fig. 2. Snapshot sequence of a space robot in response to repeated contacts with momentum dumping but without CoM control.

does not fix the inertial position of the space robot. After each impulse the robot converges to an inertial position that is farther from the target. Thus, after repeated contacts a repositioning maneuver is needed to recover the workspace. This situation is exemplified in Figs. 1 and 2 A solution to such behavior would be not only to stop the inertial drift, but also to stabilize the inertial location of the workspace while the manipulator operates. Since the maximum reachable workspace is centered at the CoM of the whole system [7], controlling this point around a suitable position would guarantee that the target object always remains in the reachable workspace while the manipulator operates. Additionally, this 
would result in a fuel efficient strategy. In fact, for all robot operations not involving contacts, the consumption of the CoM control would ideally be zero. When contacts happen, fuel would be consumed only to stop the inertial drift and restore the proper CoM location, leaving the floating-base behavior of the system unaltered.

In this paper, the momentum approach in [3] is further extended to additionally stabilize the workspace around a desired inertial location in an efficient way. First, the triangular momentum-effector dynamics in [3] is developed to further detail, then a new unified controller is derived to simultaneously regulate the end-effector, dump any accumulated angular momentum, and stabilize the CoM location. Finally, the controller is validated via experiments on a facility consisting of a redundant torque-controlled robot mounted on a satellite robotic simulator. The paper is structured as follows: Section $\mathrm{II}$ introduces the notation and the main space robot equations. Section III derives a triangular form for the CoM, momentum and end-effector dynamics. Section IV presents the proposed controller and addresses the stability. The experimental results are given in Section $\mathrm{V}$.

\section{Preliminaries}

\section{A. Problem statement}

A serial-link space robot composed of $n+1$ bodies is considered, where $n$ is the number of joints of the arm. A generalized force $\boldsymbol{w}_{b} \in \mathbb{R}^{6}$ is exerted on the robot base by satellite actuators. No orbital or environmental disturbances are considered, because they are considerably less than the actuation forces.

The operational scenario is one of capturing or manipulating a target object in orbit in the presence of motion or geometrical uncertainties. In this situation, accidental or voluntary contact may occur between end-effector and target. This would transfer momentum and accordingly create a drift between the robot and the target which would compromise the operations. Assuming that the target object is stationary in the inertial space, the problem is to command the robot end-effector to reach a desired pose in the inertial space while simultaneously ensuring no drift and maintaining a favorable relative position between robot and target.

\section{B. Main notation}

The body frame of the satellite around its CoM is denoted by $\mathcal{B}, \mathcal{E}$ is the end-effector frame, $\mathcal{T}$ is a target frame fixed in the inertial space, and $\mathcal{C}$ is a frame with nonrotating axes placed on the CoM of the whole system. We use the so-called Adjoint transformation [8], which is defined as

$$
\boldsymbol{A}_{x y}=\left[\begin{array}{cc}
\boldsymbol{R}_{x y} & {\left[\boldsymbol{p}_{x y}\right]^{\wedge} \boldsymbol{R}_{x y}} \\
\mathbf{0} & \boldsymbol{R}_{x y}
\end{array}\right] \in \mathbb{R}^{6 \times 6},
$$

where $\boldsymbol{p}_{x y} \in \mathbb{R}^{3}$ and $\boldsymbol{R}_{x y} \in \mathbb{R}^{3 \times 3}$ generically indicate the position vector and the rotation matrix from a frame $\mathcal{X}$ to a frame $\mathcal{Y}$, respectively. The operator $[\cdot]^{\wedge}$ indicates the skewsymmetric matrix of the vector argument. The symbols $\mathbf{0}$ and $\boldsymbol{E}$ are respectively the zero and identity matrices of suitable dimensions.

\section{Kinematics and dynamics}

The dynamics of the space robot [2], [9] is described by

$$
\begin{gathered}
\underbrace{\left[\begin{array}{ccc}
\boldsymbol{M}_{t} & \boldsymbol{M}_{t r} & \boldsymbol{M}_{t m} \\
\boldsymbol{M}_{t r}^{T} & \boldsymbol{M}_{r} & \boldsymbol{M}_{r m} \\
\boldsymbol{M}_{t m}^{T} & \boldsymbol{M}_{r m}^{T} & \boldsymbol{M}_{m}
\end{array}\right]}_{\boldsymbol{M}(\boldsymbol{q})}\left[\begin{array}{c}
\dot{\boldsymbol{v}}_{b} \\
\dot{\boldsymbol{\omega}}_{b} \\
\ddot{\boldsymbol{q}}
\end{array}\right]+ \\
+\underbrace{\left[\begin{array}{ccc}
\boldsymbol{C}_{t} & \boldsymbol{C}_{t r} & \boldsymbol{C}_{t m} \\
\boldsymbol{C}_{r t} & \boldsymbol{C}_{r} & \boldsymbol{C}_{r m} \\
\boldsymbol{C}_{m t} & \boldsymbol{C}_{m r} & \boldsymbol{M}_{m}
\end{array}\right]}_{\boldsymbol{C}\left(\boldsymbol{q}, \boldsymbol{v}_{b}, \boldsymbol{\omega}_{b}, \dot{\boldsymbol{q}}\right)}\left[\begin{array}{c}
\boldsymbol{v}_{b} \\
\boldsymbol{\omega}_{b} \\
\dot{\boldsymbol{q}}
\end{array}\right]=\left[\begin{array}{c}
\boldsymbol{f}_{b} \\
\boldsymbol{\tau}_{b} \\
\boldsymbol{\tau}
\end{array}\right]
\end{gathered}
$$

where $\boldsymbol{v}_{b}, \boldsymbol{\omega}_{b} \in \mathbb{R}^{3}$ are the base linear and angular velocity in body frame; $\boldsymbol{q} \in \mathbb{T}^{n}$ are the joint angles $1, \dot{\boldsymbol{q}} \in \mathbb{R}^{n}$ are the joint velocities; $\boldsymbol{f}_{b}, \boldsymbol{\tau}_{b} \in \mathbb{R}^{3}$ are the base force and torque in body frame; $\boldsymbol{\tau} \in \mathbb{R}^{n}$ are the joint torques; $\boldsymbol{M}_{m} \in \mathbb{R}^{n \times n}$ is the inertia matrix of the manipulator; and $\boldsymbol{M}_{t} \in \mathbb{R}^{3 \times 3}$, $\boldsymbol{M}_{t r} \in \mathbb{R}^{3 \times 3}, \boldsymbol{M}_{t m} \in \mathbb{R}^{3 \times n}, \boldsymbol{M}_{r m} \in \mathbb{R}^{3 \times n}$ are defined in Appendix VII.

The end-effector body velocity $\nu_{e} \in \mathbb{R}^{6}$ is given by

$$
\boldsymbol{\nu}_{e}=\boldsymbol{A}_{e b}(\boldsymbol{q})\left[\begin{array}{c}
\boldsymbol{v}_{b} \\
\boldsymbol{\omega}_{b}
\end{array}\right]+\boldsymbol{J}_{m}(\boldsymbol{q}) \dot{\boldsymbol{q}},
$$

where $\boldsymbol{J}_{m}(\boldsymbol{q}) \in \mathbb{R}^{6 \times n}$ is the manipulator Jacobian. Henceforth the functional dependence is dropped out. Denoting with $\boldsymbol{h}_{b} \in \mathbb{R}^{6}$ the total generalized momentum around $\mathcal{B}$, this can be written as

$$
\boldsymbol{h}_{b}=\underbrace{\left[\begin{array}{cc}
\boldsymbol{M}_{t} & \boldsymbol{M}_{t r} \\
\boldsymbol{M}_{t r}^{T} & \boldsymbol{M}_{r}
\end{array}\right]}_{\boldsymbol{M}_{b}}\left[\begin{array}{c}
\boldsymbol{v}_{b} \\
\boldsymbol{\omega}_{b}
\end{array}\right]+\underbrace{\left[\begin{array}{l}
\boldsymbol{M}_{t m} \\
\boldsymbol{M}_{r m}
\end{array}\right]}_{\boldsymbol{M}_{b m}} \dot{\boldsymbol{q}}
$$

The total momentum $\boldsymbol{h} \in \mathbb{R}^{6}$ around $\mathcal{C}$ is then found as $\boldsymbol{h}=\boldsymbol{A}_{c b}^{-T} \boldsymbol{h}_{b}$, which results in

$$
\boldsymbol{h}=\left[\begin{array}{c}
\boldsymbol{h}_{t} \\
\boldsymbol{h}_{r}
\end{array}\right]=\left[\begin{array}{ccc}
m \boldsymbol{R}_{c b} & -m \boldsymbol{R}_{c b}\left[\boldsymbol{p}_{b c}\right]^{\wedge} & m \boldsymbol{R}_{c b} \overline{\boldsymbol{J}}_{v} \\
\mathbf{0} & \boldsymbol{I}_{c} \boldsymbol{R}_{c b} & \boldsymbol{I}_{c} \boldsymbol{R}_{c b} \overline{\boldsymbol{J}}_{\omega}
\end{array}\right]\left[\begin{array}{c}
\boldsymbol{v}_{b} \\
\boldsymbol{\omega}_{b} \\
\dot{\boldsymbol{q}}
\end{array}\right]_{(5)}
$$

where $\boldsymbol{p}_{b c}$ and $\overline{\boldsymbol{J}}_{v}, \overline{\boldsymbol{J}}_{\omega} \in \mathbb{R}^{3 \times n}$ are given in Appendix VII and where $m, \boldsymbol{I}_{c}$ are respectively the mass and the rotational inertia around $\mathcal{C}$ of the whole body. In the following section a task space is defined and an advantageous task space dynamics is obtained thanks to the inherent decoupling properties of the momentum.

\section{TRIANGULAR DYNAMICS}

In [3] the motion of the floating multibody system was decomposed into an internal end-effector motion due to the joints and an external motion of the whole system floating in the inertial space. This was done by introducing the concept of end-effector internal velocity

$$
\boldsymbol{\nu}_{e, i n t}=\boldsymbol{J}_{m}^{*} \dot{\boldsymbol{q}} \in \mathbb{R}^{6}
$$

where $\boldsymbol{J}_{m}^{*}=\boldsymbol{J}_{m}-\boldsymbol{A}_{e b} \boldsymbol{M}_{b}^{-1} \boldsymbol{M}_{b m} \in \mathbb{R}^{6 \times n}$ is the wellknown generalized Jacobian of the end-effector. Inserting

$$
{ }^{1} \mathbb{T}^{n}=\underbrace{\mathbb{S} \times \cdots \times \mathbb{S}}_{n} \text { is the n-torus. }
$$


$\boldsymbol{h}=\boldsymbol{A}_{c b}^{-T} \boldsymbol{h}_{b}$ and (4) into (3) and further using (6), the absolute end-effector velocity can be expressed as

$$
\boldsymbol{\nu}_{e}=\boldsymbol{\nu}_{e, i n t}+\boldsymbol{A}_{e b} \boldsymbol{M}_{b}^{-1} \boldsymbol{A}_{c b}^{T} \boldsymbol{h} .
$$

Notice that in the literature $\boldsymbol{h}=\mathbf{0}$ is normally assumed, leading to $\boldsymbol{\nu}_{e}=\boldsymbol{\nu}_{e, \text { int }}$. However, here $\boldsymbol{h} \neq \mathbf{0}$, so in general $\nu_{e} \neq \nu_{e, i n t}$.

Let us assume now for the sake of simplicity and without loss of generality that the manipulator is nonredundant, i.e. $n=6$. In [3] it was shown that transforming the dynamics (2) to a new task space $\boldsymbol{v}_{\text {new }}=\left[\begin{array}{ll}\boldsymbol{h}^{T} & \boldsymbol{\nu}_{e, \text { int }}^{T}\end{array}\right]^{T}$, the resulting momentum/effector dynamics can be put in triangular form:

$$
\begin{aligned}
& \dot{\boldsymbol{h}}=\boldsymbol{w}_{c}, \\
& \boldsymbol{M}_{e}^{*} \dot{\boldsymbol{\nu}}_{e, i n t}+\boldsymbol{C}_{e}^{*} \boldsymbol{\nu}_{e, i n t}+\boldsymbol{C}_{e h} \boldsymbol{h}=\boldsymbol{w}_{e, i n t},
\end{aligned}
$$

where $\boldsymbol{M}_{e}^{*}=\left(\boldsymbol{J}_{m}^{*} \boldsymbol{M}_{m}^{*-1} \boldsymbol{J}_{m}^{* T}\right)^{-1} \in \mathbb{R}^{6 \times 6}$ with $\boldsymbol{M}_{m}^{*}=$ $\boldsymbol{M}_{m}-\boldsymbol{M}_{b m}^{T} \boldsymbol{M}_{b}^{-1} \boldsymbol{M}_{b m} \in \mathbb{R}^{n \times n}$, and where $\boldsymbol{w}_{c} \in \mathbb{R}^{6}$, $\boldsymbol{w}_{e, \text { int }} \in \mathbb{R}^{6}$ are new control input $\mathrm{S}^{2}$ namely the net centroidal wrench and end-effector internal wrench, respectively. This form has the special property of enabling the design of controllers in a cascade fashion, using $\boldsymbol{w}_{c}$ to stabilize the global motion and $\boldsymbol{w}_{e, \text { int }}$ to control the end-effector. Here we aim at developing a similar cascade structure to control the CoM, to stabilize the net angular motion and to control the end-effector. Toward this end, the same procedure as in [3] will be applied to obtain a triangular form using a new task space $\boldsymbol{v}_{\text {new }}=\left[\begin{array}{lll}\boldsymbol{v}_{c}^{T} & \boldsymbol{h}_{r}^{T} & \boldsymbol{\nu}_{e, \text { int }}^{T}\end{array}\right]^{T}$.

\section{A. CoM, angular momentum end-effector dynamics}

The CoM velocity is related to the linear momentum by

$$
\boldsymbol{v}_{c}=\frac{1}{m} \boldsymbol{h}_{t} .
$$

Based on (9), a transformation $\boldsymbol{\Gamma} \in \mathbb{R}^{12 \times(6+n)}$ can defined as

$$
\left[\begin{array}{c}
\boldsymbol{v}_{c} \\
\boldsymbol{h}_{r} \\
\boldsymbol{\nu}_{e, \text { int }}
\end{array}\right]=\underbrace{\left[\begin{array}{ccc}
\boldsymbol{R}_{c b} & -\boldsymbol{R}_{c b}\left[\boldsymbol{p}_{b c}\right]^{\wedge} & \boldsymbol{R}_{c b} \overline{\boldsymbol{J}}_{v} \\
\mathbf{0} & \boldsymbol{I}_{\mathcal{C}} \boldsymbol{R}_{c b} & \boldsymbol{I}_{\mathcal{C}} \boldsymbol{R}_{c b} \overline{\boldsymbol{J}}_{\omega} \\
\mathbf{0} & \mathbf{0} & \boldsymbol{J}_{m}^{*}
\end{array}\right]}_{\boldsymbol{\Gamma}}\left[\begin{array}{c}
\boldsymbol{v}_{b} \\
\boldsymbol{\omega}_{b} \\
\dot{\boldsymbol{q}}
\end{array}\right],
$$

where (5), (6) and (9) have been used. The generalized forces transform as

$$
\left[\begin{array}{c}
\boldsymbol{f}_{b} \\
\boldsymbol{\tau}_{b} \\
\boldsymbol{\tau}
\end{array}\right]=\boldsymbol{\Gamma}^{T}\left[\begin{array}{c}
\boldsymbol{f}_{c} \\
\boldsymbol{a}_{r} \\
\boldsymbol{w}_{e, \text { int }}
\end{array}\right],
$$

where $\boldsymbol{f}_{c} \in \mathbb{R}^{3}, \boldsymbol{a}_{r} \in \mathbb{R}^{3}, \boldsymbol{w}_{e, \text { int }} \in \mathbb{R}^{6}$ are the new control inputs, dual respectively to $\boldsymbol{v}_{c}, \boldsymbol{h}_{r}$ and $\boldsymbol{\nu}_{e, \text { int }}$. More precisely, $\boldsymbol{f}_{c}$ is the total centroidal force, $\boldsymbol{a}_{r}$ is the angular acceleration of the whole system and $\boldsymbol{w}_{e, \text { int }}$ is the end-effector internal wrench. The equation of motion (2) can be transformed to the new state [10],[11] by using an energy-preserving coordinate

\footnotetext{
${ }^{2}$ Strictly speaking, in [3] the global acceleration $\boldsymbol{a}_{h}$ was equivalently used instead of $\boldsymbol{w}_{c}$. They are related by $\boldsymbol{a}_{h}=\boldsymbol{M}_{c}^{-1} \boldsymbol{w}_{c}$.
}

transformation. For nonsingular $\boldsymbol{J}_{m}^{*}$ it is possible to invert (10) and transform (2) as:

$$
\begin{aligned}
& {\left[\begin{array}{ccc}
m \boldsymbol{E} & \mathbf{0} & \mathbf{0} \\
\mathbf{0} & \boldsymbol{I}_{\mathcal{C}}^{-1} & \mathbf{0} \\
\mathbf{0} & \mathbf{0} & \boldsymbol{M}_{e}^{*}
\end{array}\right]\left[\begin{array}{c}
\dot{\boldsymbol{v}}_{c} \\
\boldsymbol{h}_{r} \\
\dot{\boldsymbol{\nu}}_{e, i n t}
\end{array}\right]+} \\
& \quad+\left[\begin{array}{ccc}
\mathbf{0} & -\boldsymbol{C}_{r c}^{T} & -\boldsymbol{C}_{e c}^{T} \\
\boldsymbol{C}_{r c} & \boldsymbol{C}_{r} & -\boldsymbol{C}_{e r}^{T} \\
\boldsymbol{C}_{e c} & \boldsymbol{C}_{e r} & \boldsymbol{C}_{e}^{*}
\end{array}\right]\left[\begin{array}{c}
\boldsymbol{v}_{c} \\
\boldsymbol{h}_{r} \\
\boldsymbol{\nu}_{e, i n t}
\end{array}\right]
\end{aligned}
$$

Notice that the end-effector equation is inertially decoupled from the rest of the system thanks to the use of $\boldsymbol{\nu}_{e, \text { int }}$. With no surprise, notice also that the centroid and the angular momentum are also inertially decoupled, because $\boldsymbol{h}_{r}$ is around $\mathcal{C}$.

Further simplifications can be done by considering that the Coriolis and centrifugal vector terms can be shown to be identically zero for the centroid and angular momentum equations [3], simplifying (12) to

$$
\begin{aligned}
m \dot{\boldsymbol{v}}_{c} & =\boldsymbol{f}_{c}, \\
\dot{\boldsymbol{h}}_{r} & =\boldsymbol{\tau}_{c}, \\
\boldsymbol{M}_{e}^{*} \dot{\boldsymbol{\nu}}_{e, \text { int }}+\boldsymbol{C}_{e}^{*} \boldsymbol{\nu}_{e, i n t}+\boldsymbol{C}_{e c} \boldsymbol{v}_{c}+\boldsymbol{C}_{e r} \boldsymbol{h}_{r} & =\boldsymbol{w}_{e, \text { int }},
\end{aligned}
$$

where the quantity $\boldsymbol{\tau}_{c} \in \mathbb{R}^{3}$ is the net torque around the centroid, obtained as $\boldsymbol{a}_{r}=\boldsymbol{I}_{\mathcal{C}}^{-1} \boldsymbol{\tau}_{c}$. By exploiting the triangular structure it is possible to design controllers in a cascade fashion, using $\tau_{c}$ to stabilize the global angular motion and $\boldsymbol{w}_{e, \text { int }}$ to control the end-effector. Finally, the input $\boldsymbol{f}_{c}$ can be used independently to design a CoM controller.

Although in (13a), (13b) we recover the classical conservation of momentum principle, the novelty of the formulation lies instead in (13c). Indeed, the machinery (10), (11), (12) used to obtain 13 has the advantage of straightforwardly yielding the property $3^{3}$

$$
\boldsymbol{\nu}_{e, \text { int }}^{T}\left(\dot{\boldsymbol{M}}_{e}^{*}-2 \boldsymbol{C}_{e}^{*}\right) \boldsymbol{\nu}_{e, i n t}=\mathbf{0} \quad \forall \boldsymbol{\nu}_{e, \text { int }} \in \mathbb{R}^{6} .
$$

Last but not least, it suggests the use of $\boldsymbol{w}_{e, \text { int }}$ for control, resulting in better actuator coordination, as highlighted in Section IV-B. The next section shows how to design a cascade controller for CoM, angular momentum and endeffector and prove its stability.

\section{CONTROL}

The end-effector task consists in regulating the endeffector frame $\mathcal{E}$ around a desired pose $\mathcal{T}$, which is fixed in the inertial space. Let us define the error $\tilde{\boldsymbol{x}}_{e} \in \mathbb{R}^{6}$ between the frames $\mathcal{E}$ and $\mathcal{T}$ as [12]

$$
\tilde{\boldsymbol{x}}_{e}=\left[\begin{array}{c}
\boldsymbol{p}_{e t} \\
2 \boldsymbol{\epsilon}
\end{array}\right],
$$

\footnotetext{
${ }^{3}$ Since the passivity holds [9] for $\boldsymbol{M}, \boldsymbol{C}$ in [2], it also holds for the transformed matrices [12, Lemma 3.2] in [12 and for every submatrix on the diagonal.
} 
being $\epsilon \in \mathbb{R}^{3}$ is the vector part of the quaternion $\psi=\left[\begin{array}{l}\epsilon \\ \eta\end{array}\right]$ extracted from the rotation matrix $\boldsymbol{R}_{e t}$, and let $\eta$ be the scalar part. The time derivative $\dot{\tilde{\boldsymbol{x}}}_{e}$ can be expressed as

$$
\dot{\tilde{\boldsymbol{x}}}_{e}=\boldsymbol{J}_{\tilde{x}_{e} \nu_{e}} \boldsymbol{\nu}_{e},
$$

where $\boldsymbol{J}_{\tilde{x}_{e} \nu_{e}} \in \mathbb{R}^{6 \times 6}$ is the so-called coordinates representation Jacobian and is defined by [13, p.140]

$$
\boldsymbol{J}_{\tilde{x}_{e} \nu_{e}}=\left[\begin{array}{cc}
\boldsymbol{E} & \mathbf{0} \\
\mathbf{0} & \eta \boldsymbol{E}-[\boldsymbol{\epsilon}] \wedge
\end{array}\right] .
$$

The end-effector controller can be defined as

$$
\boldsymbol{w}_{e, i n t}=-\boldsymbol{J}_{\tilde{x}_{e} \nu_{e}}^{T} \boldsymbol{K}_{e} \tilde{\boldsymbol{x}}_{e}-\boldsymbol{D}_{e} \boldsymbol{\nu}_{e},
$$

where $\boldsymbol{K}_{e} \in \mathbb{R}^{6 \times 6}$ is a symmetric positive definite stiffness matrix and $\boldsymbol{D}_{e} \in \mathbb{R}^{6 \times 6}$ is a positive definite damping matrix. The CoM task is defined similarly. Given respectively the current and the desired centroidal position in the inertial frame $\boldsymbol{p}_{c}, \boldsymbol{p}_{c_{d}} \in \mathbb{R}^{3}$, the centroidal error is defined as $\tilde{\boldsymbol{p}}_{c}=\boldsymbol{p}_{c}-\boldsymbol{p}_{c_{d}}$. Then, the centroidal controller can be defined as

$$
\boldsymbol{f}_{c}=-\boldsymbol{K}_{c} \tilde{\boldsymbol{p}}_{c}-\boldsymbol{D}_{c} \boldsymbol{v}_{c},
$$

where $\boldsymbol{K}_{c} \in \mathbb{R}^{3 \times 3}$ is a symmetric positive definite stiffness matrix and $\boldsymbol{D}_{c} \in \mathbb{R}^{3 \times 3}$ is a positive definite damping matrix. In order to stabilize the system for an indefinite time, any accumulated angular momentum must be extracted to avoid persistent internal movements that may bring the robot to singularity or to the joint limits. Then, momentum dumping is commanded by

$$
\boldsymbol{\tau}_{c}=-\boldsymbol{D}_{h r} \boldsymbol{h}_{r},
$$

where $\boldsymbol{D}_{h} \in \mathbb{R}^{3 \times 3}$ is a positive definite momentum gain matrix. The closed-loop dynamics is obtained as follows. Let us first explicitly rewrite (7) in translational and rotational components. By algebraic manipulation using (5), 9) and (34), it is obtained

$$
\boldsymbol{\nu}_{e}=\boldsymbol{\nu}_{e, i n t}+\boldsymbol{G}_{c} \boldsymbol{v}_{c}+\boldsymbol{G}_{r} \boldsymbol{h}_{r},
$$

where $\boldsymbol{G}_{c}=\left[\begin{array}{c}\boldsymbol{R}_{e c} \\ \mathbf{0}\end{array}\right], \quad \boldsymbol{G}_{r}=\left[\begin{array}{c}{\left[\boldsymbol{p}_{e c}\right]^{\wedge} \boldsymbol{R}_{e c} \boldsymbol{I}_{c}^{-1}} \\ \boldsymbol{R}_{e c} \boldsymbol{I}_{c}^{-1}\end{array}\right] \in \mathbb{R}^{6 \times 3}$. The resulting closed-loop dynamics is obtained by inserting (18), (19), 20), 21) into (13), inserting (21) into (16), and inverting (6), as:

$$
\begin{aligned}
& m \ddot{\tilde{\boldsymbol{p}}}_{c}+\boldsymbol{D}_{c} \dot{\tilde{\boldsymbol{p}}}_{c}+\boldsymbol{K}_{c} \tilde{\boldsymbol{p}}_{c}=\mathbf{0} \\
& \dot{\boldsymbol{h}}_{r}+\boldsymbol{D}_{h r} \boldsymbol{h}_{r}=\mathbf{0} \\
& \boldsymbol{M}_{e}^{*} \dot{\boldsymbol{\nu}}_{e, i n t}+\boldsymbol{C}_{e}^{*} \boldsymbol{\nu}_{e, i n t}+\boldsymbol{D}_{e} \boldsymbol{\nu}_{e, i n t}+\boldsymbol{J}_{\tilde{x}_{e} \nu_{e}}^{T} \boldsymbol{K}_{e} \tilde{\boldsymbol{x}}_{e}= \\
& \quad=-\left(\boldsymbol{C}_{e c}+\boldsymbol{D}_{e} \boldsymbol{G}_{c}\right) \dot{\tilde{\boldsymbol{p}}}_{c}-\left(\boldsymbol{C}_{e r}+\boldsymbol{D}_{e} \boldsymbol{G}_{h}\right) \boldsymbol{h}_{r} \\
& \quad \dot{\tilde{\boldsymbol{x}}}_{e}=\boldsymbol{J}_{\tilde{x}_{e} \nu_{e}}\left(\boldsymbol{\nu}_{e, i n t}+\boldsymbol{G}_{c} \dot{\tilde{\boldsymbol{p}}}_{c}+\boldsymbol{G}_{r} \boldsymbol{h}_{r}\right) \\
& \dot{\boldsymbol{q}}=\boldsymbol{J}_{m}^{*-1} \boldsymbol{\nu}_{e, i n t}
\end{aligned}
$$

The state can be partitioned as $\boldsymbol{z}=\left[\begin{array}{ll}\boldsymbol{z}_{\text {whole }}^{T} & \boldsymbol{z}_{\text {arm }}^{T}\end{array}\right]^{T} \in$ $D=\mathbb{R}^{21} \times \mathbb{T}^{n}$, with $\boldsymbol{z}_{\text {whole }}=\left[\begin{array}{ccc}\tilde{\boldsymbol{p}}_{c}^{T} & \dot{\tilde{\boldsymbol{p}}}_{c}^{T} & \boldsymbol{h}_{r}^{T}\end{array}\right]^{T} \in \mathbb{R}^{9}$ and $\boldsymbol{z}_{\text {arm }}=\left[\begin{array}{lll}\boldsymbol{\nu}_{e, \text { int }}^{T} & \tilde{\boldsymbol{x}}_{e}^{T} & \boldsymbol{q}^{T}\end{array}\right]^{T} \in \mathbb{R}^{12} \times \mathbb{T}^{n}$. Then, the dynamics (22) is in the state-space form

$$
\begin{aligned}
\dot{\boldsymbol{z}}_{w h o l e} & =\boldsymbol{g}_{w}\left(\boldsymbol{z}_{\text {whole }}\right), \\
\dot{\boldsymbol{z}}_{\text {arm }} & =\boldsymbol{g}_{a}\left(\boldsymbol{z}_{\text {arm }}, \boldsymbol{z}_{\text {whole }}\right),
\end{aligned}
$$

where $\boldsymbol{g}_{w}$ is obtained from 22a,, $22 \mathrm{~b}$ ) and $\boldsymbol{g}_{a}$ from 22c (22d), 22e). Notice that the dynamics of the whole system (23a) is linear and is totally decoupled from the robot state.

\section{A. Controller stability}

The stability of the closed-loop (23) can be addressed in a cascade fashion, as follows. Let us first define a region $\Omega$ that excludes the singularities of the generalized Jacobian $\boldsymbol{J}_{m}^{*}(\boldsymbol{q})$

$$
\Omega=\left\{\boldsymbol{z} \in D: \sigma_{\min }\left(\boldsymbol{J}_{m}^{*}(\boldsymbol{q})\right)>0\right\},
$$

where $\sigma_{\min }(\ll \cdot \gg)$ is the minimum singular value of the matrix $\ll \cdot \gg$. In the region $\Omega$ the Jacobian $\boldsymbol{J}_{m}^{*}$ is welldefined together with the dynamics matrices $M_{e}^{*}, C_{e}^{*}, C_{e h}$.

Proposition IV.1. The set of equilibria $\boldsymbol{z}_{0}=\left\{\boldsymbol{z} \in \Omega: \tilde{\boldsymbol{p}}_{c}=\right.$ $\left.\dot{\tilde{\boldsymbol{p}}}_{c}=\boldsymbol{h}_{r}=\mathbf{0}, \tilde{\boldsymbol{x}}_{e}=\boldsymbol{\nu}_{e, \text { int }}=\mathbf{0}\right\}$ is asymptotically stable.

Proof. $\boldsymbol{z}_{0}$ is compact because $\mathbb{T}^{n}$ is compact. Then, cascade theorems for compact invariant sets [14] apply.

1) The systems 22a and 22b are asymptotically stable, having chosen $\boldsymbol{K}_{c}, \boldsymbol{D}_{c}$ and $\boldsymbol{D}_{h r}$ as positive definite. Therefore the whole system $\dot{\boldsymbol{z}}_{\text {whole }}=\boldsymbol{g}_{w}\left(\boldsymbol{z}_{\text {whole }}\right)$ is asymptotically stable.

2) The stability of the system $\dot{\boldsymbol{z}}_{a r m}=\boldsymbol{g}_{a}\left(\boldsymbol{z}_{a r m}, \mathbf{0}\right)$ can be addressed using the Lyapunov function

$$
V=\frac{1}{2} \boldsymbol{\nu}_{e, \text { int }}^{T} \boldsymbol{M}_{e}^{*} \boldsymbol{\nu}_{e, i n t}+\frac{1}{2} \tilde{\boldsymbol{x}}_{e}^{T} \boldsymbol{K}_{e} \tilde{\boldsymbol{x}}_{e}>0,
$$

which is always defined in $\Omega$. The time derivative along system trajectories is

$$
\begin{aligned}
& \dot{V}= \boldsymbol{\nu}_{e, \text { int }}^{T} \boldsymbol{M}_{e}^{*} \dot{\boldsymbol{\nu}}_{e, \text { int }}+\frac{1}{2} \boldsymbol{\nu}_{e, \text { int }}^{T} \dot{\boldsymbol{M}}_{e}^{*} \boldsymbol{\nu}_{e, \text { int }}+ \\
& \boldsymbol{\nu}_{e, \text { int }}^{T} \boldsymbol{J}_{\tilde{x}_{e} \nu_{e}}^{T} \boldsymbol{K}_{e} \tilde{\boldsymbol{x}}_{e}=\frac{1}{2} \boldsymbol{\nu}_{e, \text { int }}^{T}\left(\dot{\boldsymbol{M}}_{e}^{*}-2 \boldsymbol{C}_{e}^{*}\right) \boldsymbol{\nu}_{e, \text { int }}+ \\
& \quad-\boldsymbol{\nu}_{e, \text { int }}^{T} \boldsymbol{D}_{e} \boldsymbol{\nu}_{e, \text { int }}=-\boldsymbol{\nu}_{e, \text { int }}^{T} \boldsymbol{D}_{e} \boldsymbol{\nu}_{e, \text { int }} \leq 0,
\end{aligned}
$$

where (22c), 22d (22e) and (14) have been used. Applying LaSalle $\tilde{\boldsymbol{x}}_{e} \rightarrow \mathbf{0}$ is obtained and the asymptotic stability of $\dot{\boldsymbol{z}}_{a r m}=\boldsymbol{f}_{a}\left(\boldsymbol{z}_{a r m}, \mathbf{0}\right)$ is thus proven.

From 1) and 2) then follows the asymptotic stability of the closed-loop (23).

\section{B. Controller discussion}

Writing explicitly (11) and using $\boldsymbol{a}_{r}=\boldsymbol{I}_{c}^{-1} \boldsymbol{\tau}_{c}$, the actuator commands can be related to the task level controllers as:

$$
\left[\begin{array}{c}
\boldsymbol{f}_{b} \\
\boldsymbol{\tau}_{b} \\
\boldsymbol{\tau}
\end{array}\right]=\left[\begin{array}{ccc}
\boldsymbol{R}_{c b}^{T} & \mathbf{0} & \mathbf{0} \\
{\left[\boldsymbol{p}_{b c} c^{\wedge} \boldsymbol{R}_{c b}^{T}\right.} & \boldsymbol{R}_{c b}^{T} & \mathbf{0} \\
\overline{\boldsymbol{J}}_{v}{ }^{T} \boldsymbol{R}_{c b}^{T} & \overline{\boldsymbol{J}}_{\omega}{ }^{T} \boldsymbol{R}_{c b}^{T} & \boldsymbol{J}_{m}^{* T}
\end{array}\right]\left[\begin{array}{c}
\boldsymbol{f}_{c} \\
\boldsymbol{\tau}_{c} \\
\boldsymbol{w}_{e, i n t}
\end{array}\right] .
$$

In this form, the actuation distribution is seen to follow a triangular pattern. The base force $f_{b}$ is only commanded 
to control the CoM position. Conversely, the base torque $\tau_{b}$ is used to realize a net centroidal torque $\tau_{c}$ and to counterbalance the torque induced by the CoM force $\boldsymbol{f}_{c}$ due to the lever arm between $\mathcal{B}$ and $\mathcal{C}$. Finally, the joint torques are used to realize an internal end-effector wrench $\boldsymbol{w}_{e, \text { int }}$ and at the same time to counterbalance the torques induced into the joints both by $\boldsymbol{f}_{c}$ and $\boldsymbol{\tau}_{c}$. One important advantage of this triangular structure is that no end-effector force is counterbalanced by external actuators, leading to considerable fuel saving. Here, the base actuators are used only to stabilize the outer system, i.e., the motion component that strictly requires their use. This advantageous decoupling is obtained thanks to the use of $\boldsymbol{w}_{e, \text { int }}$ and does not apply when using the absolute wrench $\boldsymbol{w}_{e}$. In fact, with $\boldsymbol{w}_{e}$ the actuators would be detrimentally used to counteract the manipulator torques.

Another important feature of the proposed controller is concluded as follows. After contact, the system converges to a stationary situation in which the CoM remains fixed in the inertial space and the angular momentum is zero. Therefore, during the entire time of robot maneuvers that does not involve contact, it will remain $\tilde{\boldsymbol{p}}_{c}=\boldsymbol{v}_{c}=\boldsymbol{h}_{r}=\mathbf{0}$. In this nominal situation the controller given by the equations [18, (19), 20, 27) simply reduces to:

$$
\begin{aligned}
\boldsymbol{f}_{b} & =\mathbf{0} \\
\boldsymbol{\tau}_{b} & =\mathbf{0} \\
\boldsymbol{\tau} & =-\boldsymbol{J}_{m}^{* T}\left(\boldsymbol{J}_{\tilde{x}_{e} \nu_{e}}^{T} \boldsymbol{K}_{e} \tilde{\boldsymbol{x}}_{e}+\boldsymbol{D}_{e} \boldsymbol{\nu}_{e}\right),
\end{aligned}
$$

which is the classical transposed Jacobian free-floating controller [2]. In conclusion, with the proposed controller all the robotic operations require no fuel when no contact is involved and are performed in a free-floating fashion. External actuators are turned on only when contact occurs and their use is limited to extracting the accumulated momentum and to restoring the CoM location for the workspace need.

As a conclusive remark, note that the proposed controller 27) shares singularity properties similar to those of the transposed Jacobian free-floating controller: a) it is subject to dynamic singularities [15], b) when a singularity of $\boldsymbol{J}_{m}^{*}$ is encountered, it does not fail computationally but only results in loss of actuation in a singular direction.

\section{EXPERIMENTAL VALIDATION}

\section{A. Experimental facility description}

The CoM control strategy was validated on the On-Orbit Servicing Simulator (OOS-Sim) hardware-in-the-loop facility at the DLR [16] (see Fig. 2). The OOS-Sim is a robotic simulator for space robots, which enables testing space manipulators on ground before their actual deployment in orbit. The test manipulator is mounted on a simulator manipulator in a micro-macro configuration. The simulator manipulator reproduces the satellite's dynamics based on a real-time model integration. The test manipulator is a KUKA KR4+ lightweight robot with seven degrees of freedom. This arm is equipped with torque sensors and can be controlled both in position and torque. The simulator manipulator is a KUKA
KR120 industrial robot, which is controlled in position and has no torque sensing. The microgravity conditions in the test arm are replicated by actively compensating the joint gravity torques based on an identified model. The external forces are reconstructed by using joint torque measurements in the test arm. The simulated satellite dynamics runs on a VxWorks real-time computer at $4 \mathrm{~ms}$, whereas the space robot controller runs on a separate VxWorks real-time computer at $1 \mathrm{~ms}$. Further information regarding the hardware setup is available in [16].

With this system, the space robot controller can be simulated taking into account real dynamics, sensor noise, time delay, control discretization, and model uncertainties of the test manipulator. Conversely, the satellite dynamics simulation is model-based. The parameters of the satellite can be adapted depending on the specific scenario. In the present work, the satellite parameters are $m=150 \mathrm{~kg}, I_{x}=21.8 \mathrm{kgm}^{2}$, $I_{y}=15.0 \mathrm{kgm}^{2}, I_{z}=18.88 \mathrm{kgm}^{2}$.

\section{B. Controller implementation}

The end-effector controller in (18) requires feedback of $\tilde{\boldsymbol{x}}_{e}, \boldsymbol{\nu}_{e}$. In the experiment they are obtained from the kinematics of the simulator and test arms. The CoM and momentum controllers in (19) and 20) require feedback of $\tilde{\boldsymbol{p}}_{c}, \boldsymbol{v}_{c}, \boldsymbol{h}_{r}$. In the experiments they are obtained by using the inertia model of the robot together with the kinematics of the manipulator. In the real implementation, the required information might be estimated by fusion of the measurements from cameras (or LIDARs) and IMU.

\section{Experimental results}

Two experiments are conducted to highlight the benefit of regulating the CoM position as a means to maintain a favorable workspace location during robotic operations. To portray the situation in which the robot accidentally or voluntarily collides with a heavy target satellite, a series of impulses is given manually to its end-effector by using a rod. In a first experiment, the momentum dumping strategy in [3] is used. In this strategy, the end-effector pose is regulated around a desired inertial position while the linear and angular momenta are extracted from the system. In a second experiment, the proposed CoM control strategy (Eq. [18, (19), 20, 27) is used. Here, the additional task of restoring the proper CoM location is commanded.

To quantify the deterioration of the manipulation capability due to workspace shift, the manipulability measure in [7] is used:

$$
\text { manipulability }=\sqrt{\operatorname{det}\left(\boldsymbol{J}_{m}^{*}(\boldsymbol{q}) \boldsymbol{J}_{m}^{* T}(\boldsymbol{q})\right)}
$$

The gains used for the end-effector stiffness are $k_{e, t r a s l}=$ $1000 \mathrm{~N} \mathrm{~m}^{-1}, k_{e, r o t}=70 \mathrm{~N} \mathrm{~m} \mathrm{rad}^{-1}$. The end-effector damping gains have been designed by using the method in [12]. The gains used for the CoM control are $k_{c}=700 \mathrm{~N} \mathrm{~m}^{-1}$, $d_{c}=1080 \mathrm{~N} \mathrm{~s} \mathrm{~m}^{-1}, d_{h r}=16 \mathrm{~m}^{2} \mathrm{~s}^{-1} \mathrm{rad}^{-1}$. For the momentum dumping, equivalent gains were used $\left(d_{h t}=d_{c} / m^{2}\right)$. Fig. 3. Fig. 4 show the results for the momentum-dumping experiment. Fig. 5, Fig. 6, Fig. 7, Fig. 8 show those of the 
CoM control experiment. Additionally, the experiments can be seen in the accompanying video attachment.

1) Momentum Dumping experiment: Fig. 3 shows respectively the reconstructed external force at the end-effector, the CoM position and the manipulability measure after repeated impulses on the end-effector. Fig. 4 shows the endeffector position and the momentum. Fig. 4 shows how the momentum is successfully extracted from the system after each impulse. At the same time, the impulses induce a displacement in the CoM position, as seen in Fig. 3 . Thanks to the extraction of the accumulated momentum in the system, the CoM position converges to new stationary positions. However, after each contact the CoM is displaced farther from the initial location. Accordingly, the manipulator is in a more stretched position, in an attempt to maintain a constant inertial position. This leads to a deterioration of the workspace conditions. In particular, in Fig. 3 the manipulability is reduced after each contact, resulting in a practically singular configuration around $t_{s i n g}=140 \mathrm{sec}$. As a matter of fact, Fig. 4 further shows that at $t_{\text {sing }}$ the endeffector steady state error gets bigger after every impulse due to the low manipulability. This is explained by the fact that the low commanded torques near the singularity are counterbalanced by the static friction in the joints. In this situation, the end-effector control is lost and a repositioning maneuver of the whole system would be needed to restore the workspace.

To better visualize the workspace displacement, a series of snapshots of the experiment at $t_{0}=0 \mathrm{sec}, t_{1}=110 \mathrm{sec}, t_{2}=$ $170 \mathrm{sec}, t_{3}=220 \mathrm{sec}$ is shown in Fig. 2 Additionally, in Fig. 1 a workspace rendering at the same instants is done based on the actual experiment measurements of the satellite pose and manipulator joint. The workspace shown therein is the Maximum Reachable Workspace [7] of the robot manipulator. Fig. 1 shows that after some contacts, the target satellite exits the reachable workspace.

2) CoM Control experiment: Fig. 5 shows the reconstructed external force at the end-effector, the CoM position and the manipulability measure when the CoM control is used. First, observe in Fig. 5 that after each contact the correct CoM location is restored to the initial value. Accordingly, the manipulability measure in Fig. 5 does not drop to zero but remains in a favorable position thanks to the restored correct CoM-target location. Second, observe the end-effector position error in Fig. 6 It can be seen that, during contacts, the end-effector is displaced as a consequence of the compliance law (18). When the contacts are removed, the end-effector error converges to zero up to a precision of $1.5 \mathrm{~cm}$ due to static friction. Note that the end-effector position is restored after every contact, because no singularity is reached. Finally, note that the angular momentum in Fig. 6 is properly dumped and any rotational drift of the system is removed. The base actuator forces and torques are conclusively shown in Fig. 7. Note that the satellite forces act in reaction to the external force in Fig. 5 . In particular, as soon as one CoM displacement is generated along a direction, the satellite forces react in the same
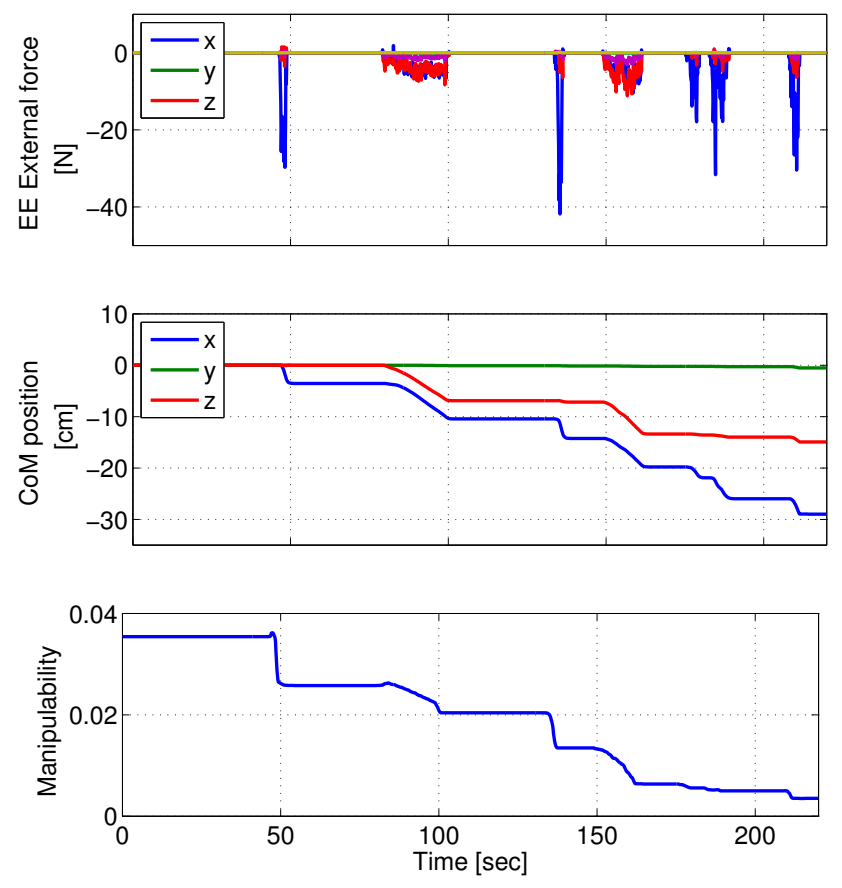

Fig. 3. Momentum Dumping Control - Workspace shift due to external contacts.
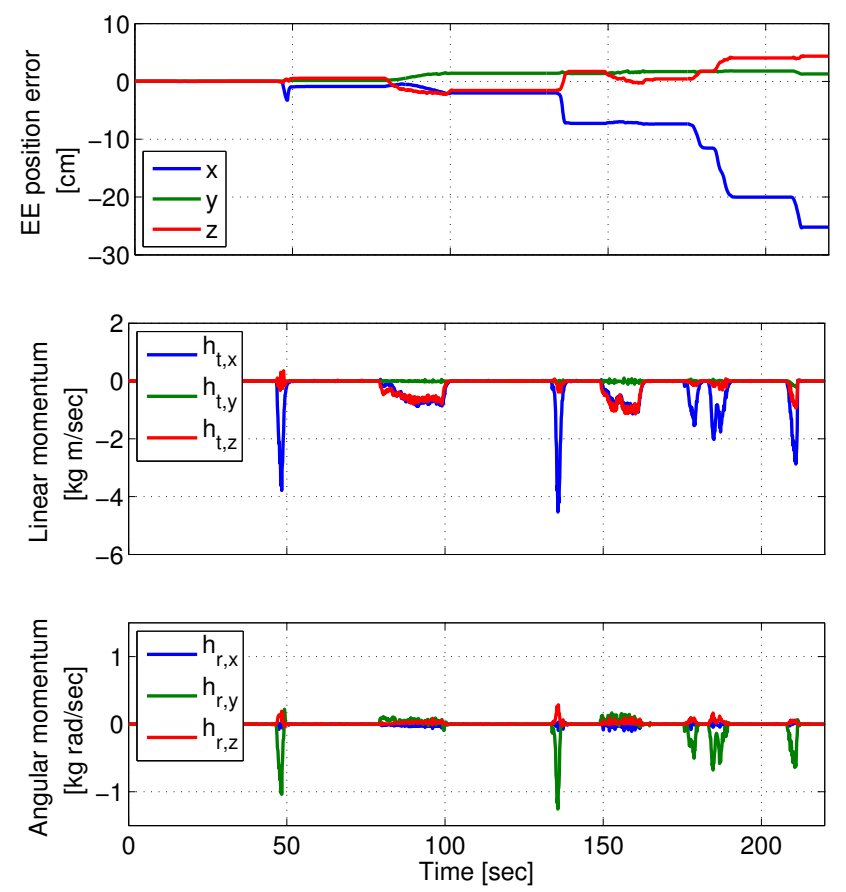

Fig. 4. Momentum Dumping Control - End effector position and momenta. 

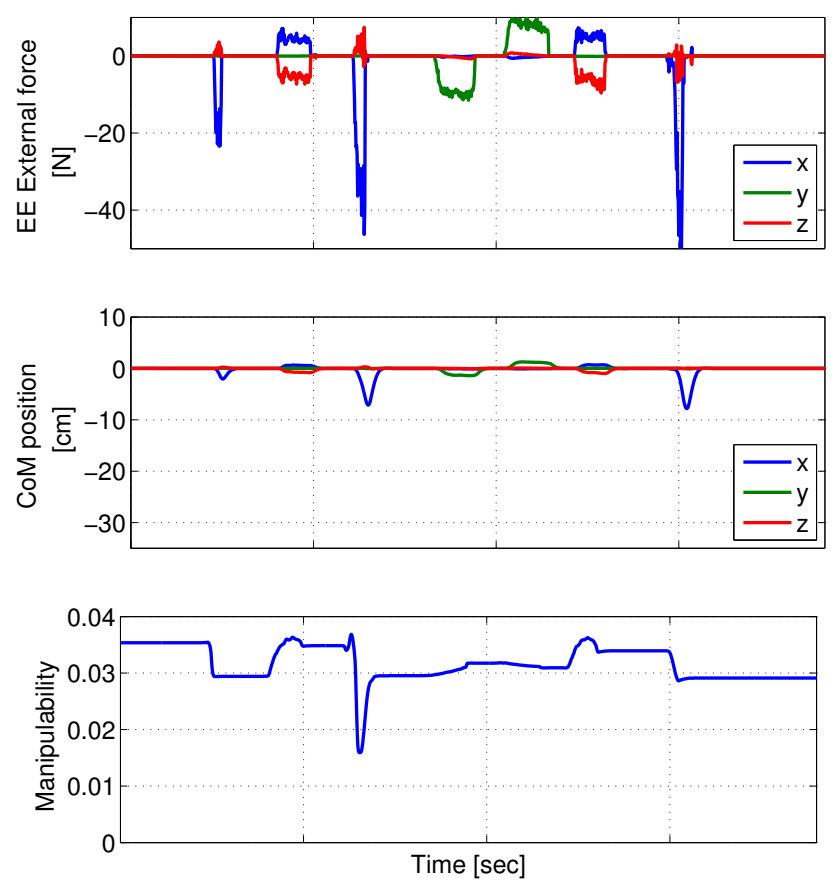

Fig. 5. CoM Control - Workspace restore after external contacts.

direction but opposite sign. For the sake of completeness, the base attitude is reported in Fig. 8. Therein it is seen that the attitude is not controlled. After each impulse, it converges to new values, which are not known in advance. The results confirm the effectiveness of the proposed control method in regulating the end-effector inertial pose, regulating the CoM location and stabilizing the rotational drift of the system induced by contacts. Furthermore, they show the usefulness of the strategy in ensuring a favorable workspace during robotic operations without the need to switch to a rigid base positioning approach.

\section{Discussion and controller limitations}

The main feature of the proposed controller is to fix the workspace without rigid positioning of the satellite. For the classical base-arm coordinated control [17], the satellite actuators persistently work against the arm's actuators. Conversely, with the proposed method the satellite is left free to move and the actuators are not used to counterbalance any manipulator force. In the absence of contact, the base actuators are automatically turned off. When contact occurs, they are used only to restore the workspace.

As previously acknowledged, a consequence of the proposed strategy is that the base attitude converges to new values after every impulse. Depending on the direction of the impulses this attitude may cancel or accumulate. In the latter case, a reorientation maneuver might be needed to avoid arm-satellite collisions or to avoid singularity. In fact, zero manipulability could still be achieved for unfavorable base attitudes. Note that the methodology developed herein does not restrain the inclusion of an additional simultaneous
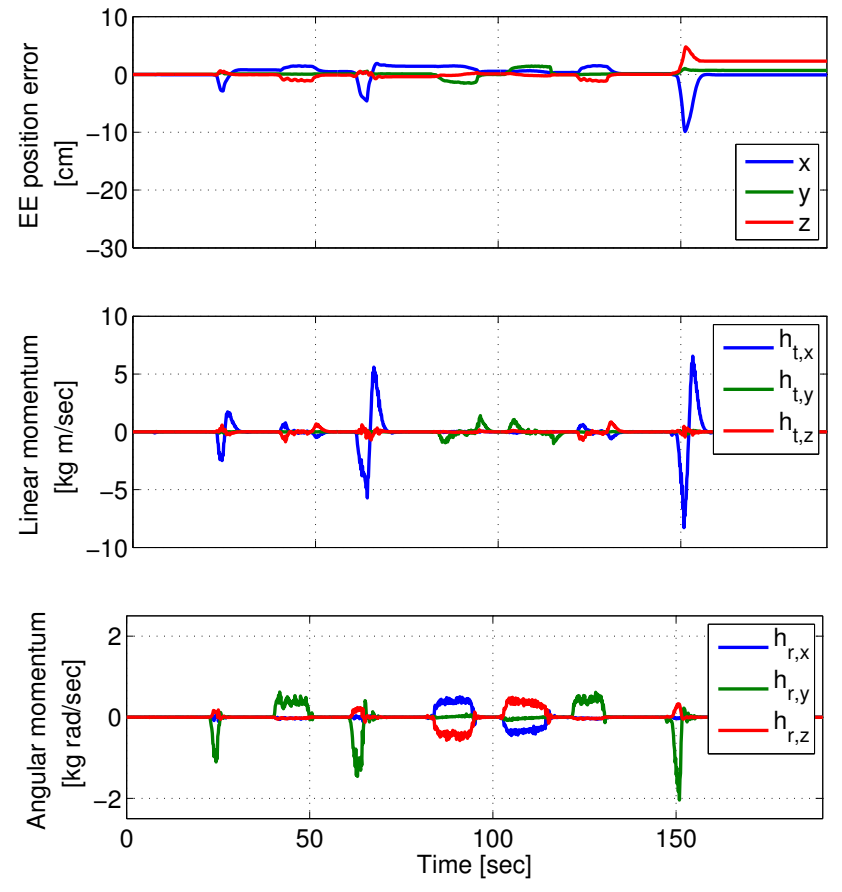

Fig. 6. CoM Control - End effector position and momenta.
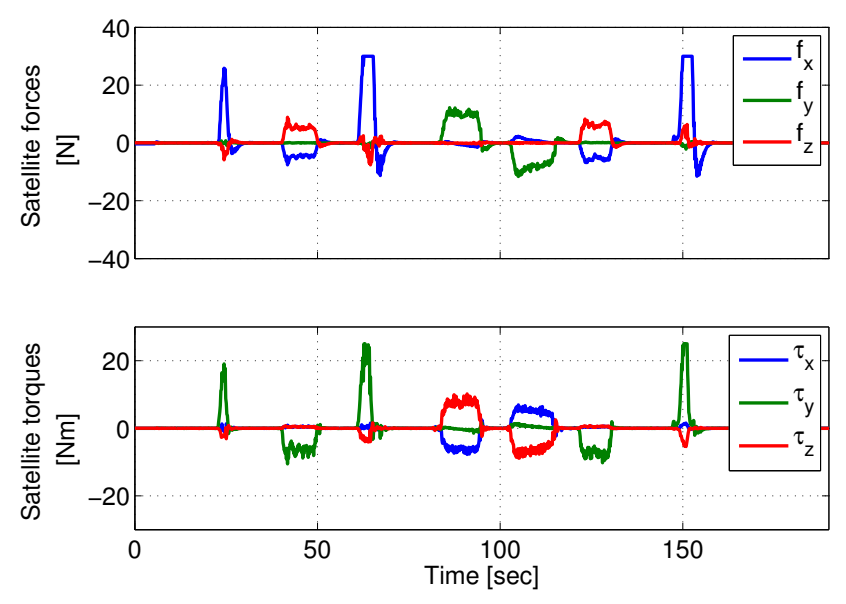

Fig. 7. CoM Control - Satellite commanded forces and torques.

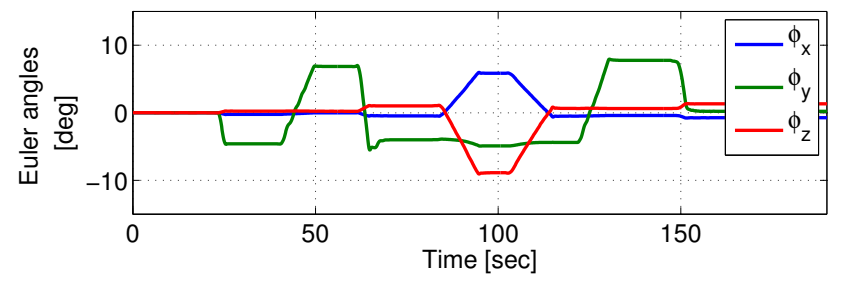

Fig. 8. CoM Control - Satellite attitude. 
reorientation task, which could be realized by exploiting the remaining three degrees of freedom in the system.

\section{CONCLUSIONS AND FUTURE WORKS}

The problem of the workspace shift due to contacts was addressed in this paper. A novel control method was derived to simultaneously restore the proper CoM location, stop the rotational drift and control the end-effector. By avoiding rigid base positioning, the base exhibits a floating behavior that leads to a reduced use of the base actuators. The control was validated with experiments.

Future work may extend the strategy to superimpose additional tasks exploiting the remaining 3DOF redundancy.

\section{APPENDIX}

\section{MOMENTUM EXPRESSION}

In terms of classical mechanics, an expression of the total momentum $\boldsymbol{h}_{b}$ around $\mathcal{B}$ is available in [2]. Here, it is reported in compact form by using a body twist formulation [8]. The momentum $\boldsymbol{h}_{i} \in \mathbb{R}^{6}$ of the body $i$ around its CoM is given by:

$$
\boldsymbol{h}_{i}=\boldsymbol{M}_{i} \boldsymbol{\nu}_{i}
$$

where $\boldsymbol{M}_{i}=\operatorname{blkdiag}\left(m_{i} \boldsymbol{E}, \boldsymbol{I}_{i}\right) \in \mathbb{R}^{6 \times 6}$ is the generalized inertia of the body $i, \boldsymbol{I}_{i}$ is its rotational inertia, $m_{i}$ is its mass and $\boldsymbol{\nu}_{i} \in \mathbb{R}^{6}$ is its body velocity. The latter can be expressed as a function of the base and joint velocities as

$$
\boldsymbol{\nu}_{i}=\boldsymbol{A}_{i b} \boldsymbol{\nu}_{b}+\boldsymbol{J}_{i} \dot{\boldsymbol{q}}
$$

being $\boldsymbol{J}_{i}=\left[\begin{array}{l}\boldsymbol{J}_{v i} \\ \boldsymbol{J}_{\omega i}\end{array}\right] \in \mathbb{R}^{6 \times n}$ the Jacobian mapping $\dot{\boldsymbol{q}}$ into $\boldsymbol{\nu}_{i}$. The total momentum of the system around $\mathcal{B}$ is given by

$$
\boldsymbol{h}_{b}=\sum_{i=0}^{n} \boldsymbol{A}_{i b}^{T} \boldsymbol{h}_{i}=\underbrace{\sum_{i=0}^{n} \boldsymbol{A}_{i b}^{T} \boldsymbol{M}_{i} \boldsymbol{A}_{i b}}_{\boldsymbol{M}_{b}}\left[\begin{array}{c}
\boldsymbol{v}_{b} \\
\boldsymbol{\omega}_{b}
\end{array}\right]+\underbrace{\sum_{i=0}^{n} \boldsymbol{A}_{i b}^{T} \boldsymbol{M}_{i} \boldsymbol{J}_{i}}_{\boldsymbol{M}_{b m}} \dot{\boldsymbol{q}} .
$$

where 30, 31 have been used. Further developing $M_{b}$ and $M_{b m}$ in 32, gives

$$
M_{b}=\left[\begin{array}{cc}
M_{t} & M_{t r} \\
M_{t r}^{T} & M_{r}
\end{array}\right], \quad M_{b m}=\left[\begin{array}{l}
M_{t m} \\
M_{r m}
\end{array}\right],
$$

where

$$
\begin{aligned}
\boldsymbol{M}_{t} & =m \boldsymbol{E} \in \mathbb{R}^{3 \times 3} \\
\boldsymbol{M}_{t r} & =-m\left[\boldsymbol{p}_{b c}\right]^{\wedge} \in \mathbb{R}^{3 \times 3} \\
\boldsymbol{M}_{r} & =\boldsymbol{I}_{b} \in \mathbb{R}^{3 \times 3} \\
\boldsymbol{M}_{t m} & =m \overline{\boldsymbol{J}}_{v} \in \mathbb{R}^{3 \times n} \\
\boldsymbol{M}_{r m} & =\sum_{i=0}^{n} \boldsymbol{R}_{i b}^{T} \boldsymbol{I}_{i} \boldsymbol{J}_{\omega, i}+m_{i}\left[\boldsymbol{p}_{b i}\right]^{\wedge} \boldsymbol{R}_{i b}^{T} \boldsymbol{J}_{v i} \in \mathbb{R}^{3 \times n}
\end{aligned}
$$

with $\boldsymbol{I}_{b}$ is the inertia of the whole system around $\mathcal{B}$ and

$$
\begin{aligned}
\boldsymbol{p}_{b c} & =\frac{1}{m} \sum_{i=0}^{n} m_{i} \boldsymbol{p}_{b i}, \\
\overline{\boldsymbol{J}}_{v} & =\frac{1}{m} \sum_{i=0}^{n} m_{i} \boldsymbol{R}_{i b}^{T} \boldsymbol{J}_{v, i} \in \mathbb{R}^{3 \times n} .
\end{aligned}
$$

The momentum around $\mathcal{C}$ is conclusively obtained as $\boldsymbol{h}=$ $\boldsymbol{A}_{c b}^{-T} \boldsymbol{h}_{b}$, which, using 32, 33, 34, results in

$$
\boldsymbol{h}=\left[\begin{array}{l}
\boldsymbol{h}_{t} \\
\boldsymbol{h}_{r}
\end{array}\right]=\left[\begin{array}{ccc}
m \boldsymbol{R}_{c b} & -m \boldsymbol{R}_{c b}\left[\boldsymbol{p}_{b c}\right]^{\wedge} & m \boldsymbol{R}_{c b} \overline{\boldsymbol{J}}_{v} \\
\mathbf{0} & \boldsymbol{I}_{\mathcal{C}} \boldsymbol{R}_{c b} & \boldsymbol{I}_{\mathcal{C}} \boldsymbol{R}_{c b} \overline{\boldsymbol{J}}_{\omega}
\end{array}\right] \boldsymbol{v}
$$

where

$$
\overline{\boldsymbol{J}}_{\omega}=\boldsymbol{R}_{c b}^{T} \boldsymbol{I}_{\mathcal{C}}^{-1} \boldsymbol{R}_{c b} \sum_{i=0}^{n} \boldsymbol{R}_{i b}^{T} \boldsymbol{I}_{i} \boldsymbol{J}_{\omega i}+m_{i}\left[\boldsymbol{p}_{b i}\right]^{\wedge}\left(\boldsymbol{J}_{v i}-\overline{\boldsymbol{J}}_{v}\right) .
$$

\section{REFERENCES}

[1] Y. Umetani and K. Yoshida, "Continuous path control of space manipulators mounted on omv," Acta Astronautica, vol. 15, no. 12, pp. 981 - 986, 1987.

[2] Y. Masutani, F. Miyazaki, and S. Arimoto, "Sensory feedback control for space manipulators," in Robotics and Automation, IEEE International Conference on, May 1989, pp. 1346-1351 vol.3.

[3] A. M. Giordano, G. Garofalo, and A. Albu-Schaffer, "Momentum dumping for space robots," in 2017 IEEE 56th Annual Conference on Decision and Control (CDC), Dec 2017, pp. 5243-5248.

[4] A. M. Giordano, G. Garofalo, M. De Stefano, C. Ott, and A. AlbuSchäffer, "Dynamics and control of a free-floating space robot in presence of nonzero linear and angular momenta," in Proceedings of the 55th IEEE Conference on Decision and Control, Dec 2016.

[5] H. Nakanishi and K. Yoshida, "Impedance Control for Free-flying Space Robots -Basic Equations and Applications-," in Intelligent Robots and Systems, 2006 IEEE/RSJ International Conference on, Oct 2006.

[6] K. Nanos and E. Papadopoulos, "On the use of free-floating space robots in the presence of angular momentum," Intelligent Service Robotics, vol. 4, no. 1, pp. 3-15, 2011.

[7] Y. Umetani and K. Yoshida, "Workspace and manipulability analysis of space manipulator," Transactions of the Society of Instrument and Control Engineers, vol. E-1, no. 1, pp. 1-8, 2001.

[8] R. M. Murray, Z. Li, and S. S. Sastry, A Mathematical Introduction to Robotic Manipulation, 1st ed. Boca Raton, FL, USA: CRC Press, Inc., 1994.

[9] G. Garofalo, C. Ott, and A. Albu-Schäffer, "On the closed form computation of the dynamic matrices and their differentiations," in Intelligent Robots and Systems (IROS), 2013 IEEE/RSJ International Conference on, Nov 2013, pp. 2364-2359.

[10] O. Khatib, "A unified approach for motion and force control of robot manipulators: The operational space formulation," Robotics and Automation, IEEE Journal of, vol. 3, no. 1, pp. 43-53, February 1987.

[11] L. Sentis and O. Khatib, "Control of free-floating humanoid robots through task prioritization," in Proceedings of the 2005 IEEE International Conference on Robotics and Automation, April 2005.

[12] C. Ott, Cartesian impedance control of redundant and flexible-joint robots. Springer, 2008.

[13] B. Siciliano, L. Sciavicco, L. Villani, and G. Oriolo, Robotics: Modelling, Planning and Control. Springer, 2009.

[14] M. I. El-Hawwary and M. Maggiore, "Reduction theorems for stability of closed sets with application to backstepping control design," Automatica, vol. 49, no. 1, pp. $214-222,2013$.

[15] E. Papadopoulos and S. Dubowsky, "Dynamic singularities in freefloating space manipulators," ASME J. Dyn.Syst., Meas., Contr.,115:1, vol. 115, pp. 44-52, 1993.

[16] J. Artigas, M. D. Stefano, W. Rackl, R. Lampariello, B. Brunner, W. Bertleff, R. Burger, O. Porges, A. Giordano, C. Borst, and A. AlbuSchaeffer, "The OOS-SIM: An on-ground simulation facility for on-orbit servicing robotic operations," in 2015 IEEE International Conference on Robotics and Automation (ICRA), May 2015.

[17] E. Papadopoulos and S. Dubowsky, "Coordinated manipulator/spacecraft motion control for space robotic systems," in Proceedings. 1991 IEEE International Conference on Robotics and Automation, Apr 1991, pp. 1696-1701 vol.2. 